Journal of the Magnetics Society of Japan Vol. 15 Supplement, No. S2 (1991)

(C) 1991 by The Magnetics Society of Japan

\title{
A STUDY OF TWO-IN-ONE TYPE HEAD FOR PERPENDICULAR RECORDING
}

Yu Heng ZHAO

Southwest Institute of Applied Magnetics Mian yang 105, Sichuan, P.R. CHINA

Abstracts... In this paper, a Two-in-0ne type composite head design for perpendicular magnetic recording is proposed. By the measurements for the recording field distributions using a large-scale model of the head, its recording resolution and sensitivity performances have been studied.

\section{INTRODUCTION}

Usually, in a recording proccess of perpendicular magnetic recording with a single pole type (SPT) head, the head-mediun spacing loss is presented on the two hands. The first is that recording sensitivity decreases with the increasing of the spacing distance (drec) between SPT head and the recording medium, because at the same time the amplitude of the recording magnetic field in recording medium will decrease, when a constant recording magnetomotive force is induced from the head, so the magnetomotive force must be raised to record the medium to magnetization saturation. The second of the spacing loss is that the recording resolution reduces with the increasing of (drec). Namely, in that case, the trailing edge in the field distribution is extended, and the width of the recording magnetization transition in the medium will expand. That overwide magnetization transition will bring the following disadvantages: (1) the amplitude of the isolated reproducing voltage pulse, proportional to the magnetization gradient $(\Delta M / \Delta X)$ will decrease. (2) in high bit density recording, the level of the recording magnetization will reduce with the interference between the adjacent magnetization transitions. [1]

Therefore, in wany works the attentions were centred on reducing the head-medium spacing distance [2]. However, in this paper the focus of attentions is not reducing the spacing distance (drec), but decreasing the width of the trailing edge in the field distribution by a new head design, thus the interference between the adjacent magnetization transitions is made to weaken, and the recording density can be further increased.

The new head is named the Two-in-one ( 2 in 1) type composite head, because it can be regarded as a composite of the both one SPT head and another one additional head as shown in Fiq. 1.

The design idea of the 2 in 1 head is that in the recording layer, the field generated from the additional head is used to cancel out the extension of the trailing edge in the field distribution of the SPT head, so a composite field with a sharp trailing edge can be obtained. To investigate the head design, the head field distributions were measured on the large-scale head model (Fig.1). As a comparison the SPT head model was also measured.

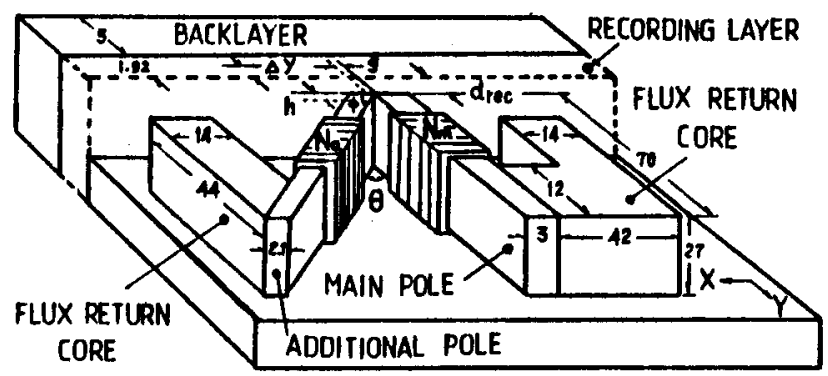

Fig. 1 Large-scale 2 in 1 head model. 


\section{EXPERIMENTAL METHOD}

The construction of the large-scale 2 in 1 type head model is shown in Fig.1. The material of the main pole is permalloy. The additional pole is composed with 6 pieces of silicon steel sheet overlapped but not bonded, in order to easily change the shape of the additional pole by moving the sheets when the angle $(\theta)$ included between the main pole and the additional pole is changed in the experiments. The material of the flux return cores is $\mathrm{Mn}-\mathrm{Zn}$ Ferrite. The top included angle $(\phi)$ of the additional pole is equal to $\left(90^{\circ}-\theta\right)$. Its tip is in quasi-contact or contact with the main pole. Windings $\mathrm{Nm}$ wrapped on the main pole and windings $\mathrm{Na}$ wrapped on the additional pole consist of 200 turns and 170 turns of copper enameled wire of $0.5 \mathrm{~mm}$ diameter, respectively. Magnetizing currents (In) and $(I a)$ for the two windings are supplied by two way separated direct supplies. The directions of (Im). and (Ia) must make the fields magnetized by the can cancel out each other as expected.

The medium simulated with a large-scale mediun model is the conventional double layer perpendicular recording media. The model of the high permeability backlayer is a non-oving pure iron plate parallel to the front surface of the main ploe. The recording layer model is an air layer which thickness equals $1.92 \mathrm{~mm}$ and it is shown in Fig. 1 by dotted line. The head model can be moved relatively to the pure iron plate in $y$-direction, so the spacing distance (drec) can be change. The flux sensing probe of Gauss-Meter used for the field measurements is fixed. The head-medium model is mounted on a stage which in $x-y$ directions is moveable with a adjustment accuracy to $0.01 \mathrm{~mm}$.

In the all measurements the values of the maximum flux density (Bmt) in the trailing edge of the recording flux density distribution at $y=0$ is always holded to constant (But=338 Gauss) by adjusting the both of (Im) and ( $\mathrm{Ia}$ ), in order to compare and appraise the head performances on the same reference magnetization level. But also, the negative peak of the flux density is limited to 5 percent of 338 Gauss by controlling (Im) and (Ia) at the all measurements.

Moreover, the flux density distributions were aeasured for the following variables, the angle $(\theta)$ defined as above, The up-moving distance $(h)$ of the additional pole; the gap length( $g$ ) between the main pole and the additional pole as shown in Fig.1.

A large-scale SPT head model was also measured as a comparison. To ensure the comparison to precise, the SPT head model was made by moving the additional pole aside from the 2 in 1 head model.

\section{RESULTS AND DISCUSSION}

To illustrate simply why the extension of the field can be canceled out, Fig. 2 shows the flux density distributions measured in the following

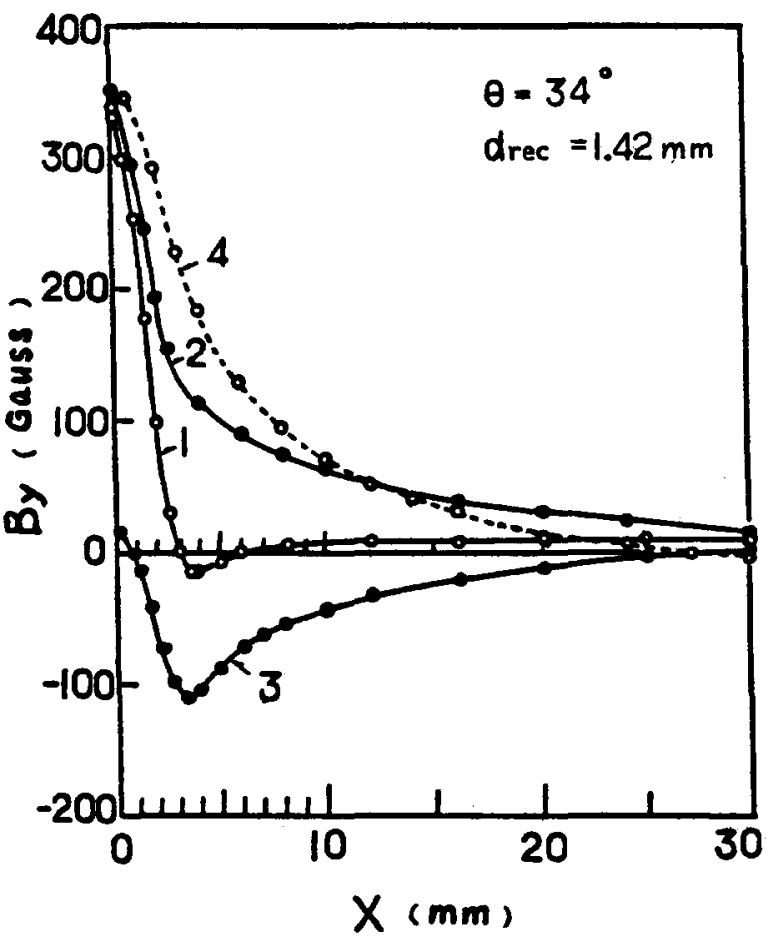

Fig. 2 Flux density distribution of both 2 in 1 head and SPT head. 

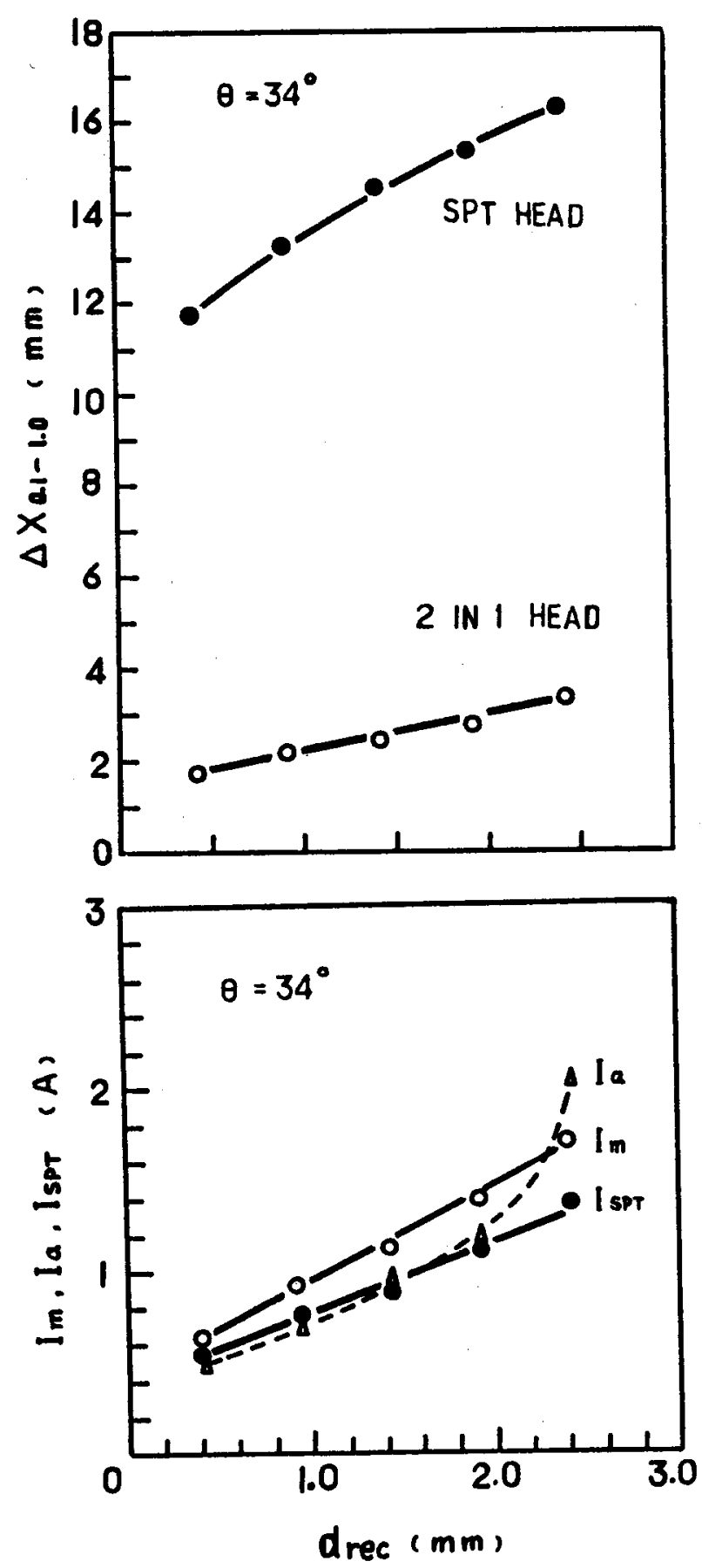

Fig. 3 relations of both $\left(\Delta X_{0.1-1.0}\right)$ and (Im), (Ia), (Ispt) to (drec). cases, in the 2 in 1 head model, (I) $1 \mathrm{~m}=1.27 \mathrm{~A}$, $I a=0.94 \mathrm{~A}$; (2) Im=1.27A, I $a=0$, (3) Im=0, I $a=0.94 \mathrm{~A}$; (4) in the SPT head model, Ispt=1.01A. The trailing edge extension of the field is canceled out by the anti-direction field magnetized by (Ia), so that, the composite field having a sharp trailing edge in the case (l) has been obtained. The sharpness of the field trailing edge of the 2 in 1 head is higher than that of the SPT head in the case (4). But, it ust be indicated that the composite field isn't directly equal to a vectorial sum of both the field in the case (2) and (3), because the fields magmetized by (Im) at $I a=0$ and $I a \neq 0$ arn't completely same with the different magnetization state of the core, and the fields magnetized by (Ia) are also similar to above.

Fig. 3 shows the relations of the trailing edge width $\left(\Delta X_{0.1-1.0}\right)$ to (drec). Although $\Delta x_{0.1-1.0}$ of the 2 in 1 head increases with the increasing of (drec), yet the trailing edge width increasing ratio $\left(\Delta X_{0.1-1.0} \Delta d r e c\right)$ is only equal to about 36 percent of the ratio of the SPT head. Besides, $\left(\Delta X_{0.1-1.0}\right)$ of the 2 in 1 head is only equal to about one fifth of $\left(\Delta x_{9-20}\right)$ of the SPT head at drec $=2.42 \mathrm{~mm}$. Even though the 2 in 1 head was measuned at the larger (drec) equal to $2.42 \mathrm{~mm}$, but the SPT head was measured at the less (drec) equal to $0.42 \mathrm{~mm}$, on the countrary, $\left(\triangle X_{0.1-1.0}\right)$ of the former is less and only equal to one third of $\left(\Delta X_{0.1-1.0}\right)$ of the latter. Above results indicate that the sharpness of the trailing edge of the 2 in 1 head field is obviously higher. Therefore, the 2 in 1 head has a higher recording resolution performance, even if at the larger (drec). So that, it can be expected that as compared with SPT head, for the same recording bit density, the permissible minium (drec) using the 2 in 1 head is larger; in other words, for the same (drec), the maximum recording bit densily using the 2 in 1 head is higher.

Fig. 3 also shows the relations of the recording currents (Im), (Ia), (Ispt) to (drec). The values of the currents increase with the 
increasing of (drec). But (Im) is larger than $(I s p t)(\operatorname{Im}=(1.2-1.25) I s p t)$. It shows that the recording sensitivity of the 2 in 1 head is lower than that of the SPT head.

The relations of $\left(\Delta X_{0.1-1.0}\right)$ to the construction factors $(\theta),(g),(h)$ of the 2 in 1 head model are shown in Fig.4.5.6. Fig. 4 shows the dependence of $\left(\Delta X_{0.1-1.0}\right)$ on the angle $(\theta)$. As will be readily seen, the optimum value
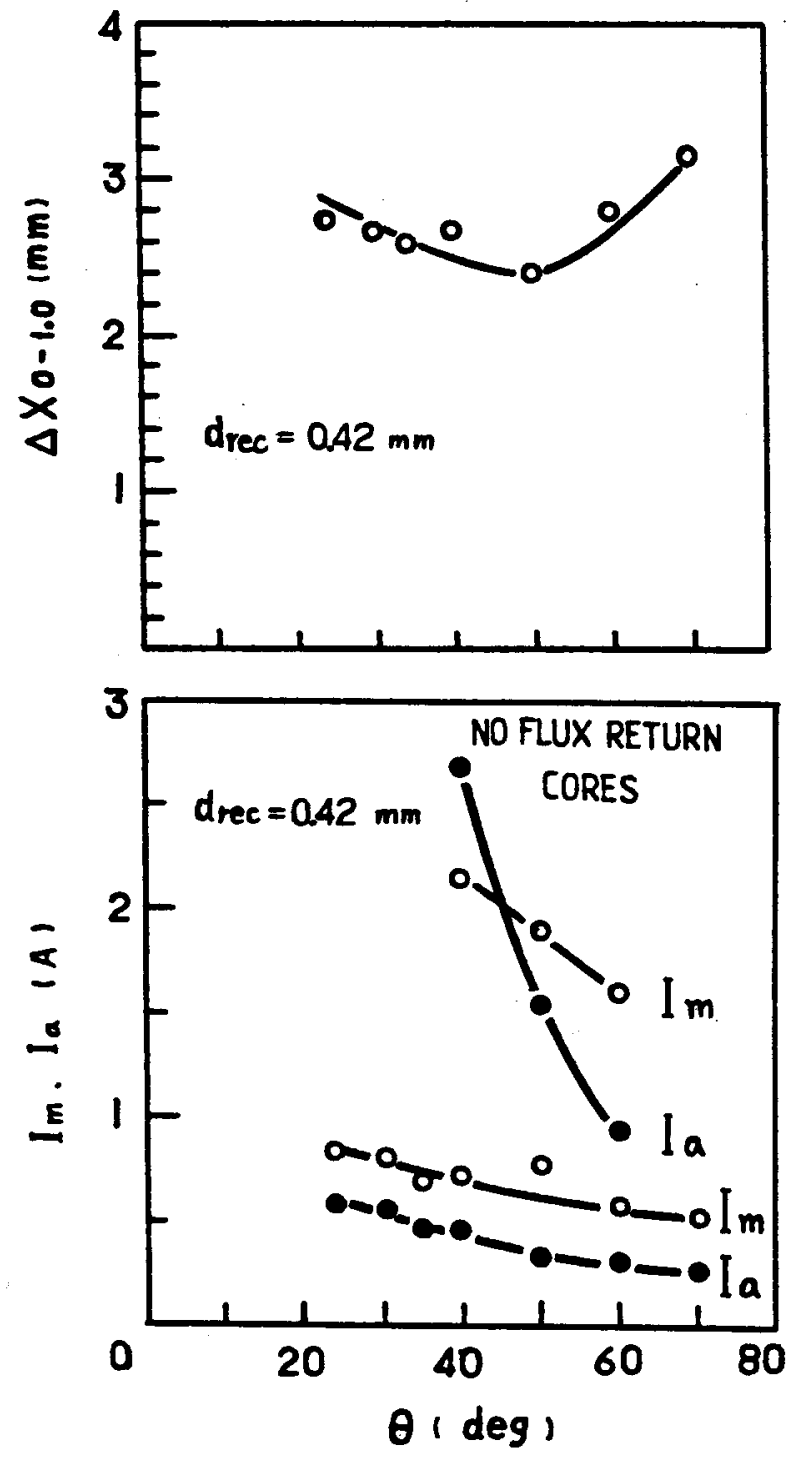

Fig. 4 relations of both $\left(\Delta X_{0-1.0}\right)$ and (Im), (Ia), (Ispt) to $(\theta)$. of $(\theta)$ making $\left(\Delta X_{0.1-1.0}\right)$ becomed minium for each (drec) has existed. For example, the optimum value of $(\theta)$ equal about $50^{\circ}$ at drec $=0.42 \mathrm{~mm}$. It is caused of the different flux distributions from the additional pole at the different $(\theta)$, so the effect canceled out the extension of the field trailing edge is also changed with them.

The relations of the recording currents (Im), (Ia), (Ispt) to $(\theta)$ is also shown in Fig. 4. The values of the currents decrease with the
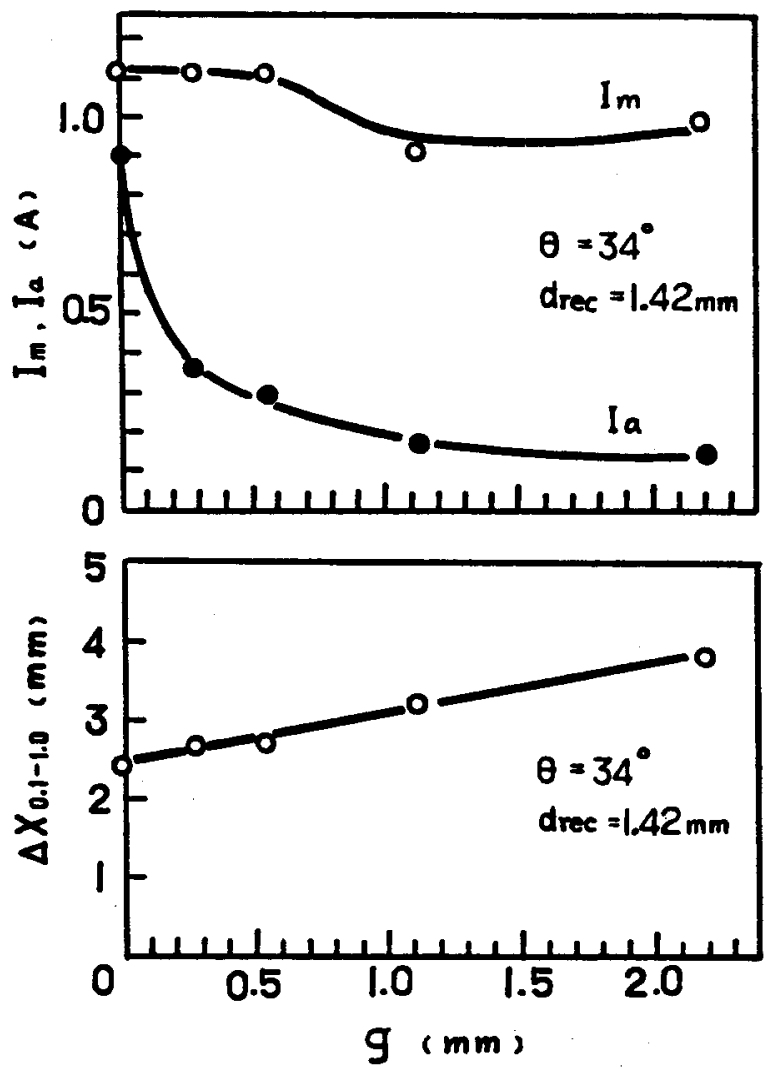

Fig. 5 relations of both $\left(\Delta \mathrm{X}_{0.1-1.0}\right)$ and (Im), (Ia), (Ispt) to (g).

increasing of $(\theta)$. Especially, in that case which the flux return cores of both the main pole and additional pote don't exist, the decreasing more obvious. It is caused of that 
interaction which the main pole and the additional pole are taken as the recording flux by-path each other, and the interaction is weakened with the increasing of $(\theta)$.

Fig. 5 shows the influences of the gap length (g) on $\left(\triangle X_{0.1-1.0}\right)$. The trailing edge width $\left(\Delta X_{0.1-1.0}\right)$ widens gradually with the increasing of $(g)$. So that, in order to obtain as sharp trailing edge as possible, (g) is expected as narrow as possible. this just is the reason why the additional pole is in quasi-contact or contact with the main pole. Even so, the problem shorted out the recording flux in the gap as the ring head shouldn't take place, because based on the magnetic circuit law, the principal part of the flux magnelized by (Im) flowes along the closed circuit (main pole $\rightarrow$ its flux return core $\rightarrow$ air gap $\rightarrow$ recording medium $\rightarrow$ main pole). For the same reason, the principal part of the flux magnetized by (Ia) shouldn't be shorted out by the main pole too.

The influences of $(g)$ on $(I m),(I a)$ are also shown in Fig.5. The increasing of (Im) with the decreasing of $(g)$ isn't obvious, and it can be look on as a proof of the above reason. However, the increasing of (la) is more obvious, but it is principaly on account of that the peak of the negative field magnetized by (Ia) shifts near the peak of the positive field magnetized by (I⿴囗十) with the decreasing of $(g)$, so the required negative field should heighten for the cancelling out. Furthermore, from Fig.6 it can be seen that at a no large $(g)$, for example at $g=2.5 \mathrm{~mm}$, $\left(\Delta X_{0.1-1.0}\right)$ whould be still less, but the decreasing of (Ia) is much.

If the 2 in 1 head pole surfaces parallel to the medium surface are weared, the gap length between the two poles will increase. Consequently, it was considered to move up the additional pole in $\mathrm{Y}$-direction, in order to avoid the wear. Fig.6 shows the relation of $\left(\Delta X_{0.11 .0}\right)$ to the up-moving distance (h) The values of $\left(\triangle X_{0.1-1.0}\right)$ are still far less than that of the SPT head.

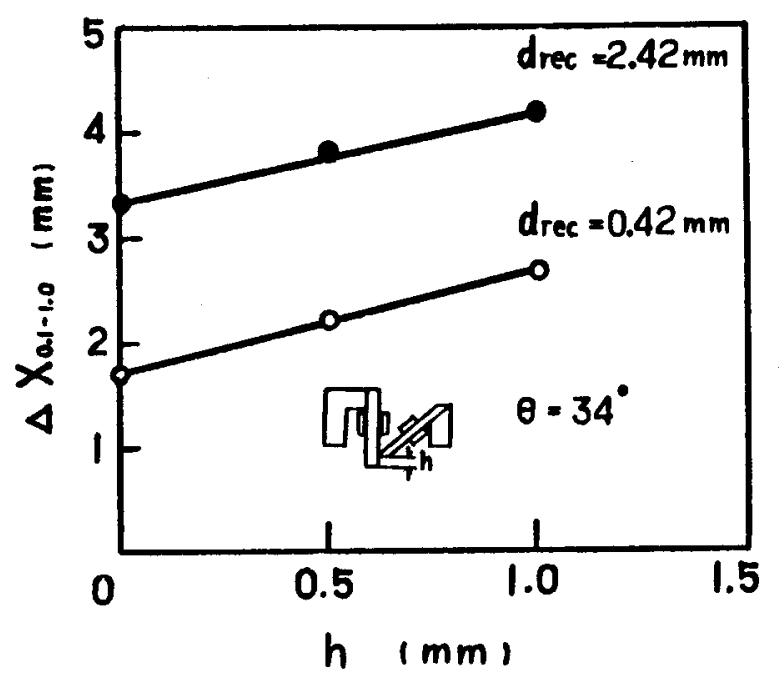

Fig. 6 relation of $\left(\Delta X_{0.1-1.0}\right)$ to $(h)$

The relation of the maximum flux density (Bmt) to the recording depth $(\Delta y)$ in the recording madiun is shown in Fig. ?. First of all, the condition which (Bmt) equals 338 Gauss at $\Delta y=0$ was made. Then, the flux density distributions were measured at the different values of $(\Delta y)$. For both the 2 in 1 head and SPT head, it is almost similar that the velues of the maximum flux density (Bmt) decrease with the increasing of $(\Delta y)$. The value of (Bmt) at $\Delta y=1.5 \mathrm{ma}$ is about 71 percent of that at $\Delta y=0$.

But also, the relation of $\left(\Delta X_{0.1-1.0}\right)$ to $(\triangle y)$ is also shown in Fig. 7. The values of $\left(\triangle X_{0.1-1.0}\right)$ of the 2 in 1 head are also less than that of the SPT head for any values of $(\triangle y)$. It clearly indicates that the recording resolution performance of the 2 in 1 head is higher, even though in the depth of the recording leyer. But the ratio $\left(\Delta X_{0.1+1.0} \Delta y\right)$ of the 2 in 1 head is higher than that of the SPT head.

\section{CONCLUSION}

The results measured by the large-scale 2 in 1 head model show that with the proper $(\theta)$, (h) 

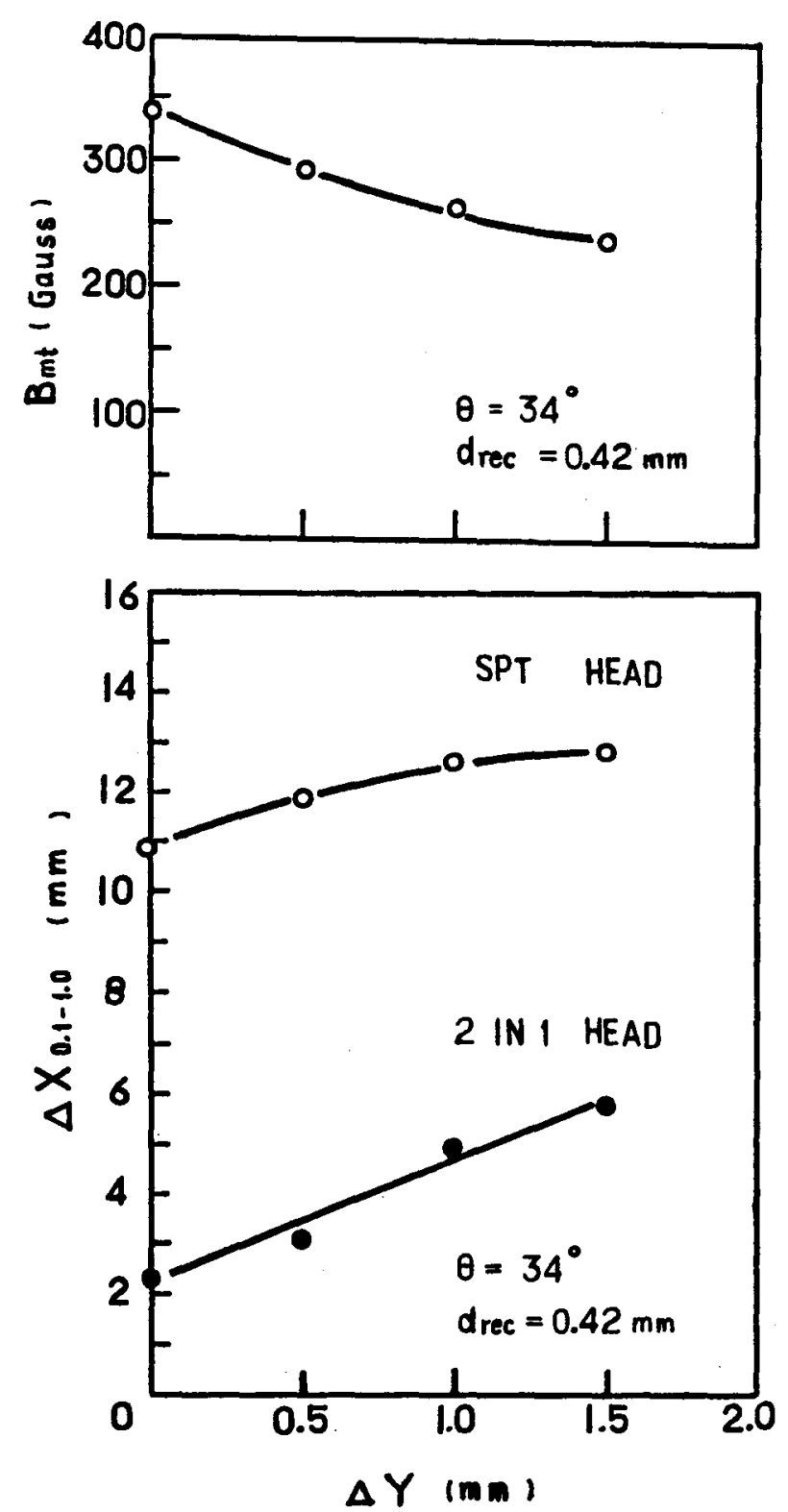

Fig. 7 relations of both (Bmt) and $\left(\Delta X_{0.1-1.0)}\right.$ to $(\Delta Y)$.

and as small ( $g$ ) as possible, the sharp field trailing edge can be obtained. This head design would be useful for improving the recording resolution at the larger (drec). And further high recording bit density should be possible by it.

\section{REFERENCES}

[1] S.Yamamoto et al, Magn. Soc Japan, "Write Spacing Loss in perpendicular Magnetic " Vol. 10, PP. 105-108, 1986.

[2] Shigenobu Sobajima, Magn. Soc Japan, "Tribology of Thin Film Media For Perpendicular Magnetic Recording" Vol.11, PP27-31, 1987. 